\title{
The effect of multivalent counterions to the structure of highly dense polystyrene sulfonate brushes
}

Jing $\mathrm{Yu}^{1,2}$, Jun Mao ${ }^{1,2}$, Guangcui Yuan ${ }^{3,4}$, Sushil Satija ${ }^{3}$, Wei Chen ${ }^{1,2 *}$, and Matthew Tirrell ${ }^{1,2 *}$

\footnotetext{
${ }^{1}$ Institute for Molecular Engineering, University of Chicago, Chicago, IL 60637

${ }^{2}$ Materials Science Division, Argonne National Laboratory, Argonne, IL 60439

${ }^{3}$ NIST Center for Neutron Research, National Institute of Standards and Technology, Gaithersburg, MD 20899-6102

${ }^{4}$ Department of Polymer Engineering, The University of Akron, Akron, Ohio 443250

* Corresponding authors.

Email:

wchen@anl.gov

mtirrell@uchicago.edu
} 


\begin{abstract}
Surface tethered polyelectrolyte brushes are scientifically interesting and technologically relevant to many applications, ranging from colloidal stabilization to responsive and tunable materials to lubrication. Many applications operate in environments containing multi-valent ions, media in which our scientific understanding is not yet well-developed. We synthesized high-density polystyrene sulfonate (PSS) brushes via surface initiated atom-transfer radical polymerization, and performed neutron reflectivity (NR) measurements to investigate and compare the effects of mono-valent $\mathrm{Rb}^{+}$and tri-valent $\mathrm{Y}^{3+}$ counterions to the structure of the densely tethered PSS brushes. Our NR results show that in mono-valent $\mathrm{RbNO}_{3}$ solution, the dense PSS brush retained its full thickness up to a salt concentration of $1 \mathrm{M}$, whereas it immediately collapsed upon adding $1.67 \mathrm{mM}$ of tri-valent $\mathrm{Y}^{3+}$. Increasing the concentration of $\mathrm{Y}^{3+}$ beyond this level did not lead to any significant further structure change of the PSS brush. Our findings demonstrate that the presence of multi-valent counterions can significantly alter the structure of polyelectrolyte brushes, in a manner different from mono-valent ions, which has implications for the functionality of the brushes.
\end{abstract}




\section{Introduction}

Polyelectrolyte brushes consist of charged polymer chains extended outward into solution with one end tethered to an interface[1]. The polyelectrolyte chains stretch away from the anchoring surface, giving rise to a brush structure and unique properties. Understanding the structure and properties of polyelectrolyte brushes under different solution environments has a significant impact in a wide range of applications, ranging from responsive and tunable materials to lubrication to drug delivery to energy storage [2-7].

Due to the complexity of combining short range steric and long range Coloumbic interactions, the structure and properties of polyelectrolyte brushes can be dramatically influenced by the surrounding solution environment, e.g., the ionic strength, $\mathrm{pH}$, and counterions in the solution $[8,9]$. For weak (so-called, annealed) polyelectrolyte brushes, the degree of ionization is a function of the $\mathrm{pH}$ [9-11], whereas brushes formed of strong (so-called, quenched) polyelectrolytes, such as polystyrene sulfonate (PSS), are not affected by the $\mathrm{pH}$, and hence are somewhat simpler model systems to study the important ion-brush interactions. Scaling models show that the thickness of polyelectrolyte brushes in low concentration mono-valent salt solutions is determined from the balance between the osmotic pressure of the counterions and the chain elasticity [12]. For relatively dense and strongly charged brushes, in low ionic strength, or the socalled osmotic regime, the thickness of the brushes is independent of the grafting density and solution ionic strength. The thickness of the brush in a theta solvent is given by:

$$
h \sim a N f^{0.5},
$$


where $N$ is the degree of polymerization of the polyelectrolyte chain, $a$ is the size of the monomer, and $f$ is the fraction of charged monomers, which in the case of strong polyelectrolytes is always very close to 1.0 .

Upon adding more salt into the solution, the brushes enter the salted brush regime, where the thickness of the brushes follows an Alexander scaling form of the brush thickness in good solvent, and the brush thickness is given by

$$
h \sim a N f^{2 / 3} \sigma^{1 / 3} C_{s}^{-1 / 3}
$$

where $\sigma$ is the grafting density and $C_{\mathrm{s}}$ is the solution salt concentration. In the salted brush regime, the brush thickness shrinks with increasing salt concentration, but only as a relatively weak power law $-1 / 3$, representing the weakening excluded volume interactions with increasing salt. The crossover of the two regimes occurs when the solution salt concentration $\mathrm{C}_{\mathrm{s}}$ equals the inner concentration of counterions $\mathrm{C}_{\mathrm{I}}$ in the brush. The scaling theory of polyelectrolyte brushes has been verified by various experimental efforts [13-15]. Surface forces apparatus (SFA) measurements using sparsely tethered polystyrene sulfonate (PSS) brushes generated from solution adsorption of hydrophilic/hydrophobic diblock polymers at the solid-water interface and X-ray reflection of poly(ethylethylene)-b-poly(styrene sulfonate) at the air-water interface have showed that the behavior of the PSS brush can be described by the scaling model in mono-valent salt solution. Tran et al carried out neutron reflectivity measurements on end-tethered PSS brushes with the degree of sulfonation ranging from $35 \%$ to $60 \%$, and found out that the brush thickness was insensitive to the concentration of $\mathrm{NaCl}$ in the solution up to $1 \mathrm{M}$ [16]. That study, however, is complicated by the low, and possibly inhomogeneous, sulfonation of polystyrene chains. The functional dependencies on $\mathrm{N}$ 
and $\mathrm{C}_{\mathrm{s}}$ have been verified experimentally [13]. One element that experimentally is not in strict accord with the ansatz of the scaling theory is the crossover point itself. It was found [13] hat transition from the osmotic to the salted brush regime required addition of only about $15 \%$ of $\mathrm{C}_{\mathrm{I}}$ to the solution. This is due to the phenomenon of counterion condensation; only $80-85 \%$ of the counterions are free to exert osmotic pressure to swell the brush. Counterion condensation is crucial to the understanding of the effects of multivalent ions [17].

Beyond scaling theory, numerous theoretical and simulation efforts have been made in order to get a more detailed picture of polyelectrolyte brush structure. Selfconsistent-field theory (SCF) and recently non-local density functional theory have been used to study polyelectrolyte brushes profiles in different ionic strength and solvent conditions [18-20]. Various simulation methods, including molecular dynamic simulation, Monte Carlo simulation and Langevin dynamics simulation, have also been applied to investigate the structures of polyelectrolyte brushes under different solution conditions [21-23].

Polyelectrolyte brushes experience an abrupt decrease in brush thickness in the presence of multi-valent counterions, analogous to the precipitation of polyelectrolytes in multi-valent ionic solutions $[24,25]$. SFA experiments have shown that adding multivalent counterions induces collapse of the PSS brushes, and adhesion force was measured when separating two PSS brushes as a result of multi-valent electrostatic bridging between chain segments $[26,27]$. This collapse of polyelectrolyte brushes is in part due to a decrease in the osmotic pressure of counterions through the replacement of multiple mono-valent counterions by multi-valent counterions, and in part due to the greater 
propensity of multi-valent ions for counterion condensation, leading to the possibility of effective electrostatic bridges. Multi-valent ions are very common in biological systems and industrial formulations, so a deep understanding of this behavior is critical for the field.

Our previous SFA experiments provided many insights in the effect of multivalent counterions to the structure of PSS brushes; however, these experiments were done with sparely tethered PSS brushes due to the limit of adsorption method used to generate the PSS brushes[26]. The structure of densely tethered PSS brushes in the presence of multi-valent counterions needs to be further explored. Recent advances in surfaceinitiated atom transfer radical polymerization (SI-ATRP) have enabled the synthesis of high grafting density polyelectrolyte brushes with very good control over the architecture of grafted chains [28]. Densely tethered PSS brushes with $100 \%$ sulfonation can be synthesized via direct polymerization of sodium 4-vinylbenzenesulfonate with wellcontrolled brush thickness using SI-ATRP [29], making it an ideal model system to study the effect of ionic strength and multi-valent counterions to the structure of polyelectrolyte brushes.

In this study, we used SI-ATRP to synthesize densely tethered PSS brushes with controlled film thickness, and probed the structure and collapse of the PSS brushes in the presence of mono-valent and tri-valent counterions in solution by neutron reflectivity (NR) measurements. Our results show that the film thickness of densely grafted PSS brushes is insensitive to the presence of mono-valent salt up to a salt concentration of $1 \mathrm{M}$ due to high grafting density but very sensitive to the presence of tri-valent yttrium cations. 


\section{Experimental section}

\subsection{Materials}

Sodium 4-vinylbenzenesulfonate (SSNa, technical, $\geq 90 \%)$, (3-aminopropyl) triethoxysilane (APTES, 99\%), $\alpha$-bromoisobutyryl bromide (BIBB, 98\%), triethylamine (TEA, $\geq 99 \%$ ), 2, 2-bipyridyl (bpy, $\geq 99 \%$ ), $\mathrm{Cu}(\mathrm{I}) \mathrm{Br}$ (99.999\%), THF (anhydrous, 99.8\%), methanol $(\geq 99.9 \%)$ were purchased from Aldrich and used without further purification. Single-sided polished silicon wafers (100-oriented, 3 inch diameter, $5 \mathrm{~mm}$ thickness) were purchased from University Wafer (Boston, MA). Deionized water was prepared from a Millipore Milli-Q system with a resistivity of $18.2 \mathrm{M} \Omega \mathrm{cm}$.

\subsection{Atomic Force Microscopy (AFM)}

A Bruker Multimode 8 atomic force microscope was used to measure the surface topography of the dry PSSNa brush film. The spring constant of the Si AFM tip used in the measurement was $2 \mathrm{~N} / \mathrm{m}$.

\subsection{Fourier-Transform Infrared}

Fourier-Transform Infrared (FT-IR) Spectroscopy was recorded using a PerkinElmer Frontier FT-IR spectrometer. One hundred and twenty-eight scans were collected from 4,000 to $800 \mathrm{~cm}^{-1}$ at a spectral resolution of $2 \mathrm{~cm}^{-1}$.

\subsection{X-ray Reflectivity}


$\mathrm{X}$ ray reflectivity measurements were conducted using a Bruker, D8 Avance Xray reflectometer employing $\mathrm{Cu} \mathrm{K \alpha}$ radiation at National Institute of Standards and Technology (NIST) Center for Neutron Research (NCNR) (Gaithersburg, MD). The copper source was operated at $40 \mathrm{kV}$ and $40 \mathrm{~mA}$, and the wavelength was $0.154 \mathrm{~nm}$. The beam width was $10 \mathrm{~mm}$ and the beam height was $0.1 \mathrm{~mm}$.

\subsection{Neutron Reflectivity}

Neutron reflectivity (NR) measurements were performed at NCNR NIST on the NG7 horizontal reflectometer using a house designed sample chamber. The reflected

intensity was measured as a function of momentum transfer vector $\left[q_{z}=\frac{4 \pi \sin (\theta)}{\lambda}\right]$, where $\theta$ is the incident angle and $\lambda=0.475 \mathrm{~nm}$ is the neutron wavelength. $\mathrm{D}_{2} \mathrm{O}$ was used in all the NR experiments. Model-dependent calculations of the reflectivity profile on basis of SCF were performed using the Abeles matrix method implemented in the Refl1D analysis package $[30,31]$.

\section{Synthesis and Characterization of PSS brushes}

3.1 Preparation of PSSNa brushes on silicon wafer via SI-ATRP.

The synthesis procedure of PSSNa brushes on silicon wafer is illustrated in Figure 1. Silicon wafers were washed with Piranha solution $\left(\mathrm{H}_{2} \mathrm{SO}_{4}: \mathrm{H}_{2} \mathrm{O}_{2}=7: 3(\mathrm{v} / \mathrm{v})\right)$ at boiling temperature for $1 \mathrm{~h}$ to remove the organic residues on the surfaces. The wafers were subsequently rinsed with an excess of deionized water and dried in a nitrogen flow.

The freshly prepared hydroxyl terminated silicon wafers were placed in a vacuum desiccator with a vial containing $2 \mathrm{~mL}$ of APTES. The desiccator was then pumped down 
to 600 mTorr, isolated from the pump and left under vacuum for $1 \mathrm{~h}$. The silicon wafers were then annealed at $110{ }^{\circ} \mathrm{C}$ for $1 \mathrm{~h}$. After annealing, a silicon wafer was immersed in 20 $\mathrm{mL}$ anhydrous THF in a petri dish. TEA $(0.9 \mathrm{~mL}, 6.3 \mathrm{mmol})$ and $\mathrm{BIBB}(0.78 \mathrm{~mL}, 6.3$ mmol) were then added into THF by syringes. After $1 \mathrm{~h}$ reaction, the silicon wafer was removed from the solution and rinsed with methanol followed by drying under a nitrogen flow.

SI-ATRP grafting of PSSNa was carried out in a glass vessel purged with nitrogen. In a typical reaction, $\mathrm{SSNa}(5 \mathrm{~g}, 24.2 \mathrm{mmol}), \mathrm{Cu}(\mathrm{I}) \mathrm{Br}(35 \mathrm{mg}, 0.24 \mathrm{mmol})$ and bpy $(75 \mathrm{mg}, 0.48 \mathrm{mmol})$ were dissolved in a 1:1 mixture of methanol and water $(50 \mathrm{~mL})$ to give a reaction solution. The reaction solution was degassed by three freeze-pumpthaw cycles and then injected into the glass vessel in which the initiator-functionalized wafer was placed. The polymerization was carried out at room temperature for $1.5 \mathrm{~h}$ and quenched by exposure to the air. The obtained PSSNa-grafted silicon wafer was removed from the solution immediately, cleaned ultrasonically in deionized water and rinsed thoroughly, and then dried under a nitrogen flow. 


\section{Silicon Wafer}

(a)

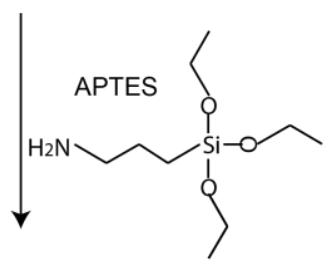

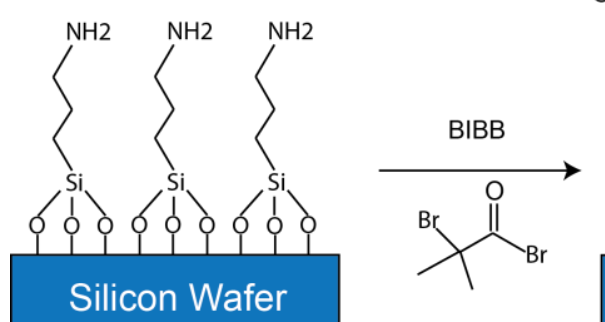

(b)

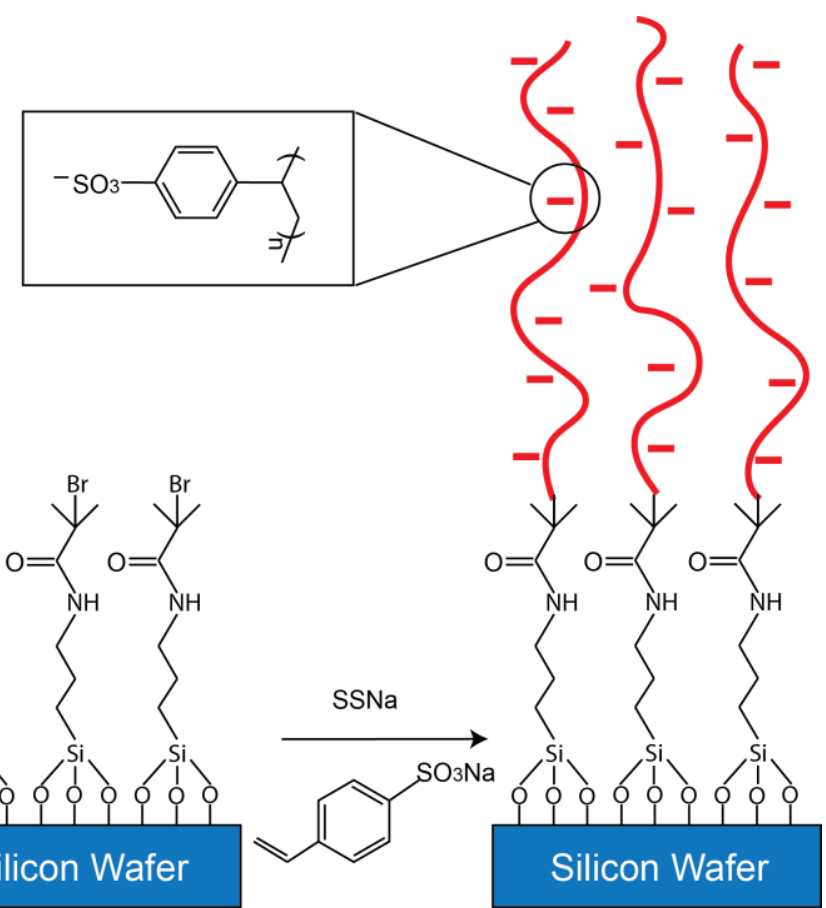

(c)

(d)

Figure 1. Schematic on the synthesis of PSSNa brush on a silicon wafer via SI-ATRP.

\subsection{Characterization of dry PSSNa films}

The surface topology of synthesized PSSNa film was measured by AFM, as shown in Figure 2 a. The RMS roughness of the PSSNa surface is $0.29 \mathrm{~nm}$, indicating the PSSNa films synthesized by SI-ATRP are fairly homogeneous. FT-IR result of PSS brush grafted on silicon wafer was presented in Figure $2 \mathrm{~b}$. The absorption at 1055 and $1023 \mathrm{~cm}^{-}$ 1 can be ascribed to characteristic peaks of $-\mathrm{SO}_{3}$ symmetric stretching vibrations, indicating the formation of the PSS brush. The peaks at 1514 and $837 \mathrm{~cm}^{-1}$ are correlated to the aromatic $-\mathrm{C}=\mathrm{C}$ - stretching vibrations and the $=\mathrm{C}-\mathrm{H}$ out of plane deformation vibrations, respectively. 
(a)

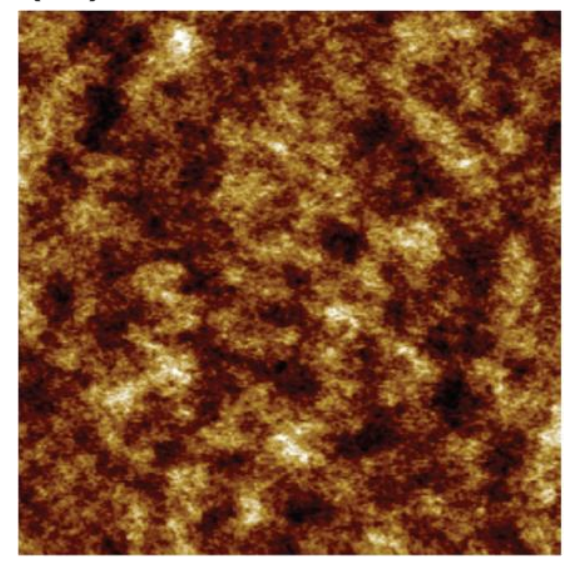

\section{$1 \mathrm{~nm}$}

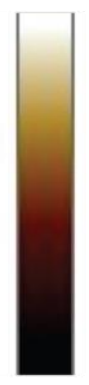

$-1 \mathrm{~nm}$

$1.6 \mu \mathrm{m}$

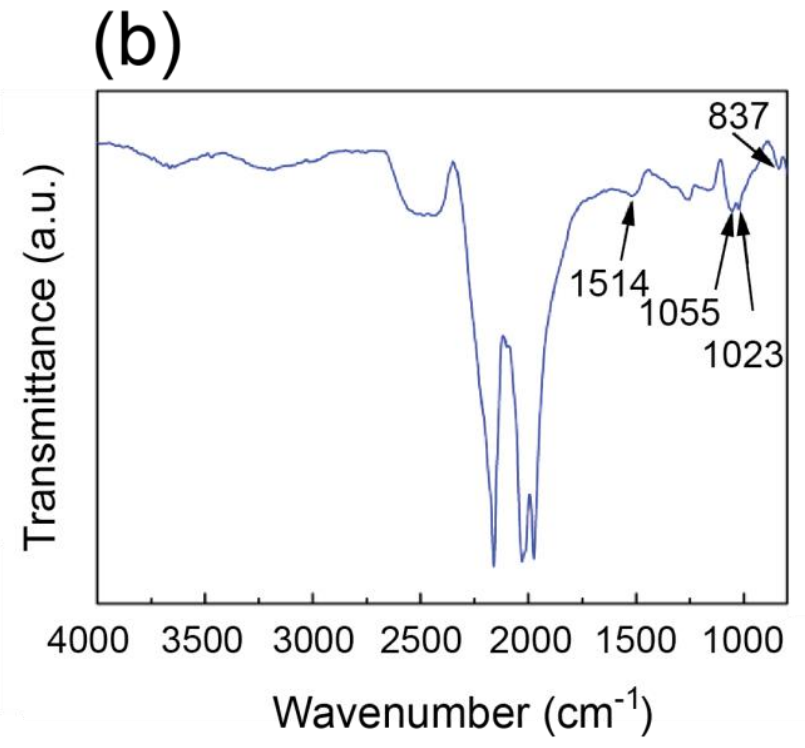

Figure 2. Characterization of dry PSSNa films synthesized via SI-ATRP. (a) AFM image of a dry PSSNa surface (b) FT-IR spectra of a PSSNa grafted Si wafer.

We used X-ray reflectivity to measure the thickness of dry PSSNa film (Figure S1). The fitting of X-ray reflectivity data was done by using a multilayer fitting algorithm with fit parameters optimized to best fit the data. The silicon wafer surface roughness is about $4 \mathrm{~nm}$ measured from x-ray reflectivity measurement on a bare silicon wafer used in 
this study. This value is set as a constant in all the fittings of our X-ray and neutron reflectivity data. For the two separately synthesized PSSNa brush surfaces used in this study, the dry film thicknesses obtained from fitting are 12.6 and $13.5 \mathrm{~nm}$ respectively, which indicates that our SI-ATRP method has very good control over the brush film thickness.

\section{Neutron reflectivity measurements}

We employed neutron reflectivity (NR) to investigate the structure of PSSNa brushes in $\mathrm{D}_{2} \mathrm{O}$ solutions with different concentrations of mono-valent salt, $\mathrm{RbNO}_{3}$, and tri-valent salt, $\mathrm{Y}\left(\mathrm{NO}_{3}\right)_{3}$. Figure 3 shows a schematic of our experimental geometry. The fitting of the PSSNa brush reflectivity data was done using Refl1D software. We used the parabolic function based upon SCF theory to fit our reflectivity data set $[19,32]$. The brush density profile is given by:

$$
\begin{gathered}
\varphi(z)=\varphi_{0}\left(1-\left(\frac{z-z_{0}}{L}\right)^{2}\right)^{p}, \\
\varphi_{\sigma}(z)=\varphi(z) \frac{e^{-0.5\left(\frac{z}{\sigma}\right)^{2}}}{\sqrt{2 \pi \sigma^{2}}}, \\
\text { and } \rho(z)=\rho_{p} \varphi_{\sigma}(z)+\rho_{s}\left(1-\varphi_{\sigma}(z)\right),
\end{gathered}
$$

where $\varphi_{0}(\mathrm{z})$ is the polymer volume fraction at the silicon interface, and $\varphi_{\sigma}(\mathrm{z})$ is the polymer volume fraction convoluted with brush roughness $\sigma . \rho(\mathrm{z}), \rho_{\mathrm{p}}$, and $\rho_{\mathrm{s}}$ are the scattering length densities of the film, PSS polymer, and solvent $\left(\mathrm{D}_{2} \mathrm{O}\right)$ respectively. The shape exponent $p$ is predicted to have a value of 1 in a good solvent and 0.5 in a theta solvent. In our fitting, we used $p=1$ and $p=0.5$ for swelled brush and collapsed brush 
respectively. The scattering length density of PSS, $\rho_{\mathrm{p}}=2.248 \times 10^{-6} / \AA^{2}$, is obtained from the fitting of the X-ray reflectivity data from dry PSSNa films.

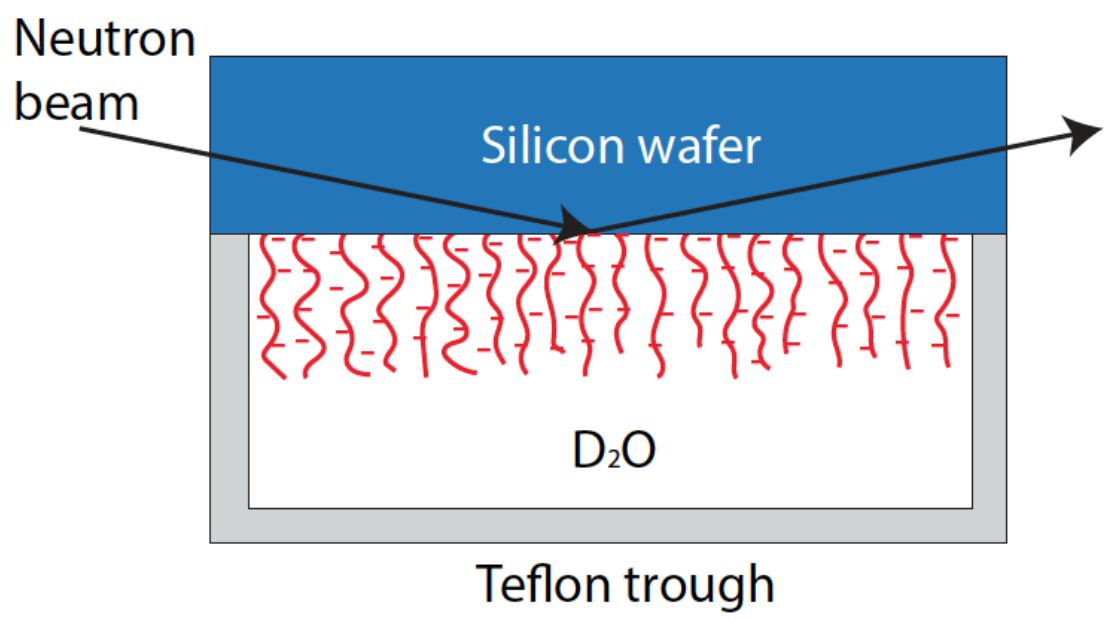

Figure 3. Schematic of the neutron reflectivity experiment setup.

The NR of PSS brushes in pure $\mathrm{D}_{2} \mathrm{O}, 1 \mathrm{mM}, 10 \mathrm{mM}, 100 \mathrm{mM}, 500 \mathrm{mM}$ and $1 \mathrm{M}$ $\mathrm{RbNO}_{3}$ solutions are shown in Figure $4 \mathrm{a}$. For all the $\mathrm{RbNO}_{3}$ concentrations investigated, there was no significant change over the NR spectra, indicating that the PSS brush stayed in the osmotic regime even in solution of $1 \mathrm{M}$ ionic strength. The high grafting density gives rise to a high osmotic pressure of the counterions, which extends the osmotic regime to higher ionic strength. In this regime, the brush thickness does not change with the ionic strength of the solution.

Figure $4 \mathrm{~b}$ shows the convoluted volume fraction profiles of the PSS brush in $\mathrm{RbNO}_{3}$ solutions. Above the silicon interface, the volume fraction profiles of the PSS brush can be well described by the CSF parabolic function with a diffused tail. This tail has its origin in the fluctuation of the brush at the outer interface [30]. The volume fraction profiles also reveal that the PSS brush in solution swells to thickness of about 
$100 \mathrm{~nm}$, which is about 8 times of its dry film thickness $(12.6 \mathrm{~nm})$. Within our fitting error, no significant changes were observed in the best-fitted volume fraction profiles of the PSS brush in solutions with different concentrations of $\mathrm{RbNO}_{3}$.
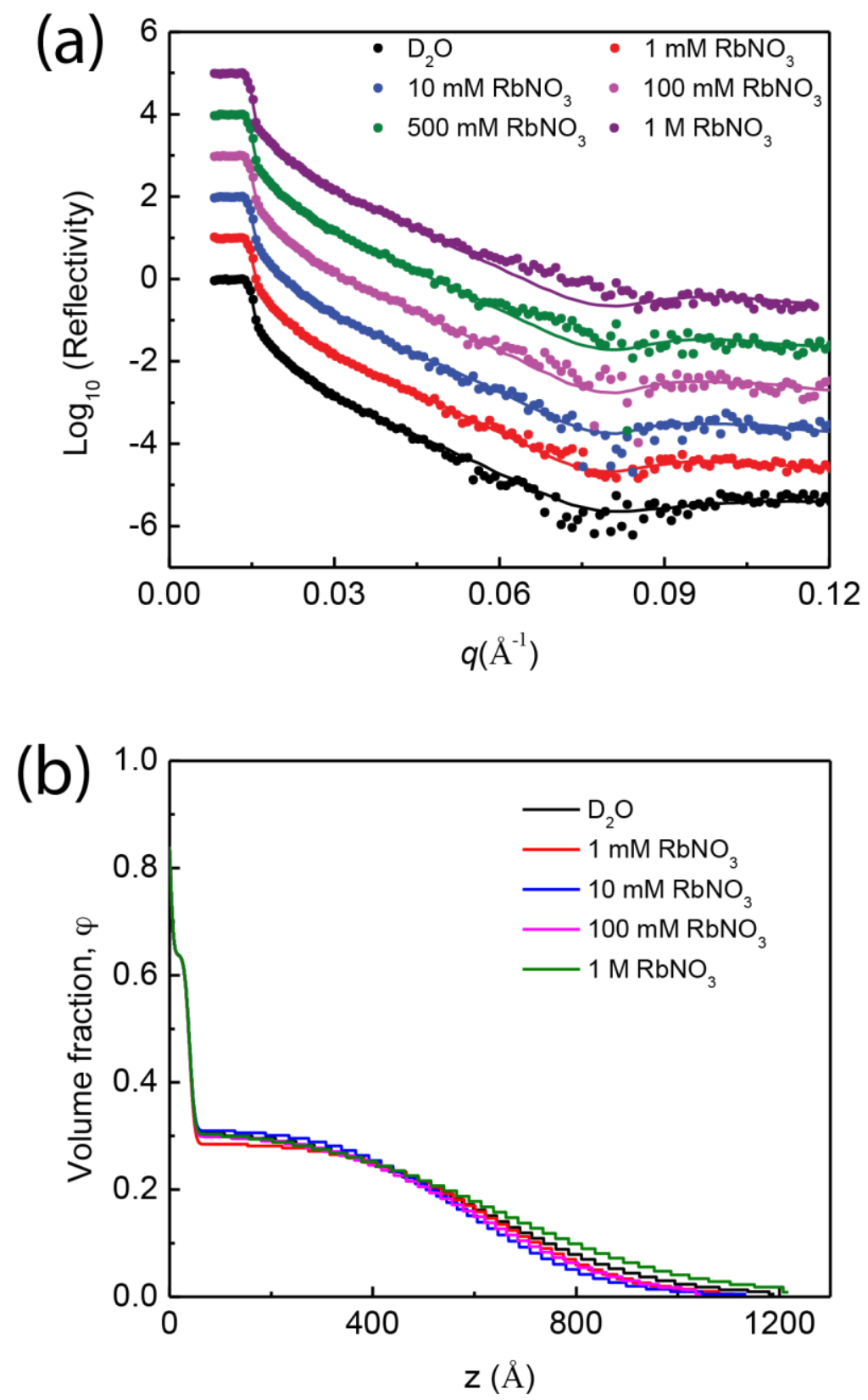

Figure 4 (a) NR of a PSS brush in mono-valent salt solutions with different $\mathrm{RbNO}_{3}$ concentrations. The reflectivity curves are shifted for the clarity of the presentation. (b) Best fitted volume fraction profiles obtained by reflectivity fitting base on equation (3)- 
(5). The small variations in the extended tail region towards the bulk solution $(z>600 \AA)$ of the profiles are within the error of our fitting.
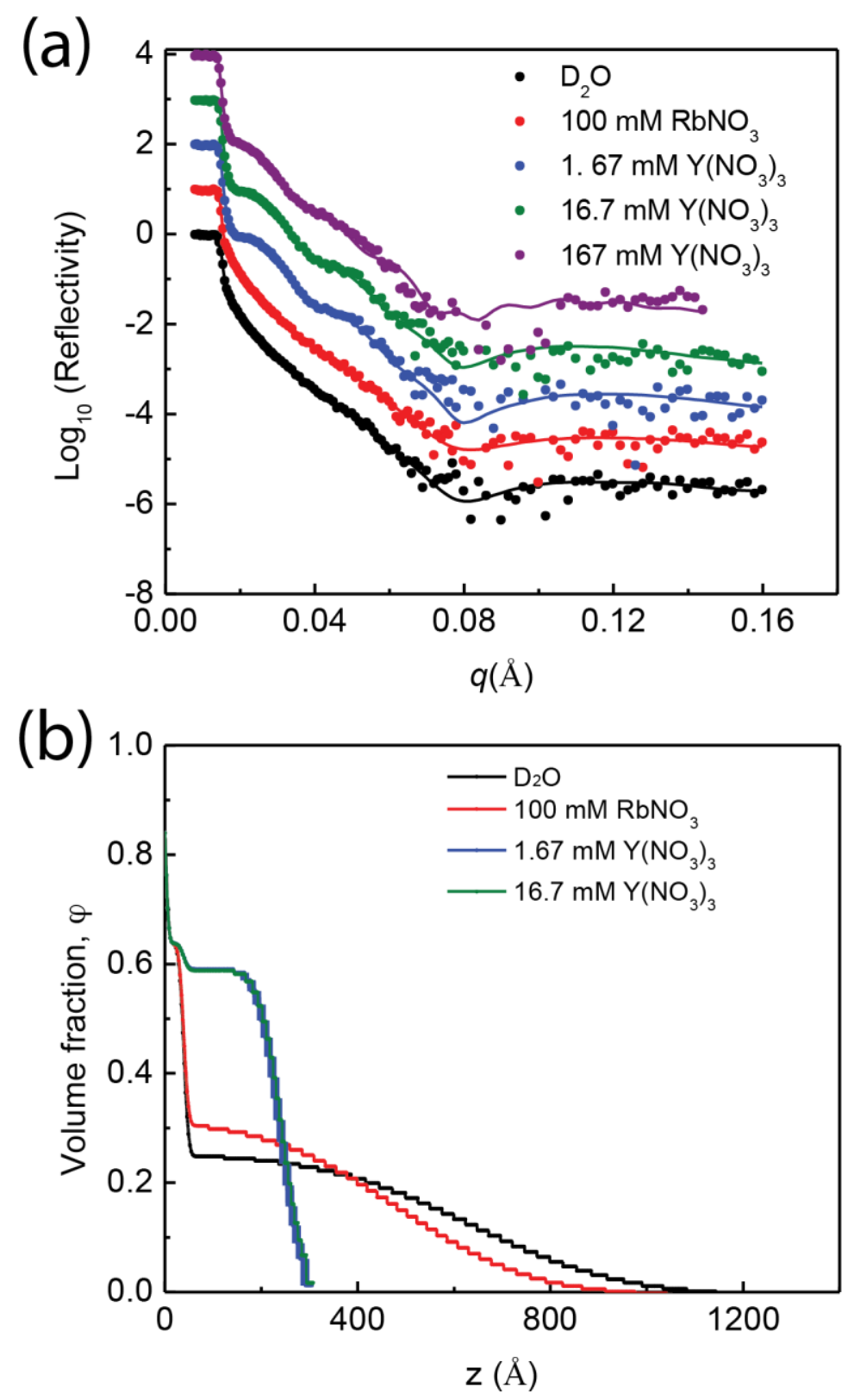

Figure 5. (a) NR of a PSS brush in water, $100 \mathrm{mM} \mathrm{RbNO}_{3}, 1.67 \mathrm{mM} \mathrm{Y}\left(\mathrm{NO}_{3}\right)_{3}, 16.7 \mathrm{mM}$ $\mathrm{Y}\left(\mathrm{NO}_{3}\right)_{3}$ and $167 \mathrm{mM} \mathrm{Y}\left(\mathrm{NO}_{3}\right)_{3}$ solutions. The reflectivity curves are shifted for the 
clarity of the presentation. (b) Volume fraction profiles of the PSS brush in different solutions.

Figure 5 shows NR of a PSS brush in the presence of tri-valent yttrium cations, $\mathrm{Y}^{3+}$. The high grafting density PSS brush, although retaining its extended brush structure up to an ionic strength of $1 \mathrm{M}$ in mono-valent $\mathrm{RbNO}_{3}$ salt solution, immediately collapsed upon adding $1.67 \mathrm{mM}$ tri-valent $\mathrm{Y}^{3+}$ cations, an equivalent ionic strength of $10 \mathrm{mM}$. Future increase the concentration of $\mathrm{Y}\left(\mathrm{NO}_{3}\right)_{3}$ to $16.7 \mathrm{mM}$ and $167 \mathrm{mM}$ did not cause any big change in NR of the PSS brush, indicating that the structure of PSS brush underwent a shape collapse in the presence of tri-valent cations. The volume fraction profiles also reveal that the PSS brush thickness underwent a first order phase transition like change, as it collapsed from $100 \mathrm{~nm}$ to about $30 \mathrm{~nm}$ upon adding $1.67 \mathrm{mM} \mathrm{Y} \mathrm{Y}^{3+}$ cations, and retained the same thickness at higher concentrations of $\mathrm{Y}^{3+}$.

Both theoretical and experimental work has shown that adding multi-valent counterions can cause an abrupt change in the structure of polyelectrolyte brushes [17, $25,27]$. We have previously shown that the existence of tri-valent cations (e.g., $\mathrm{La}^{3+}$ and $\left.\mathrm{Ru}\left(\mathrm{NH}_{3}\right)_{6}{ }^{3+}\right)$, even at very low concentration, can cause abrupt collapse of sparsely tethered PSS brushes $[26,27]$. Our NR results demonstrate that this conclusion also holds for densely tethered PSS brushes. Previous studies have focused on contributions to the brush collapse from a decrease in the osmotic pressure of the counterions[23]. Furthermore tri-valent ions bring three charges into the brush, while condensing only one ion [17]. The entropy gain from releasing three mono-valent $\mathrm{Rb}^{+}$cations to the solution when the PSS brush takes up one tri-valent $\mathrm{Y}^{3+}$ cation drives the ion exchange process. 
As the tri-valent $\mathrm{Y}^{3+}$ counterions replacing the confined mono-valent $\mathrm{Rb}^{+}$ions in the PSS brush, the osmotic pressure of the counterion is significantly reduced, leading to the shrinking of the brush layer. While providing an important contribution to lower brush thickness, the osmotic pressure difference is insufficient to fully explain brush behaviors in the presence of multi-valent ions, such as adhesion between two brush-coated surfaces measured in our previous SFA experiments and the observed first order transition from extended to collapsed state of polyelectrolyte brushes in this study. Very recently, Brettmann et al developed a phenomenological mean-field approach with a model that specifically includes bridging of the polyelectrolyte chains by the multiple charges on the multi-valent counterions[17]. The model showed that increasing the concentration of multi-valent ions leads to a sharp collapse of the polyelectrolyte brush thickness.

\section{Discussion}

The grafting density is of great importance to the structure of polyelectrolyte brushes in salt solution. It determines the boundary between the osmotic regime and the salted brush regime, and therefore affects the response of the brush layer to the change of solution ionic environment. In previous SFA studies using sparely tethered PSS brushes, the osmotic to salted brush regime transition happened at an ionic strength of $0.01 \mathrm{M}$ [14]. The PSS brushes used in this study are much denser, which significantly extends the osmotic regime as the transition from the osmotic regime to salted brush regime did not happen at an ionic strength of $1 \mathrm{M}$.

The interaction between two polyelectrolyte brushes strongly depends on the structure of the brushes. A stable brush structure is essential to many applications of 
polyelectrolyte brushes, such as stabilizing colloidal particles and providing good lubrication. Our results show that the PSS brushes generated from SI-ATRP have fairly high grafting density, and can retain a fully extended brush structure at much higher mono-valent salt concentration in comparison to PSS brushes generated through solution adsorption (Figure 6a), and therefore maintain its functions across a wider range of solution ionic environment. However, the existence of multi-valent counterions can cause abrupt collapse of the brushes (Figure 6b).

(a) Brush

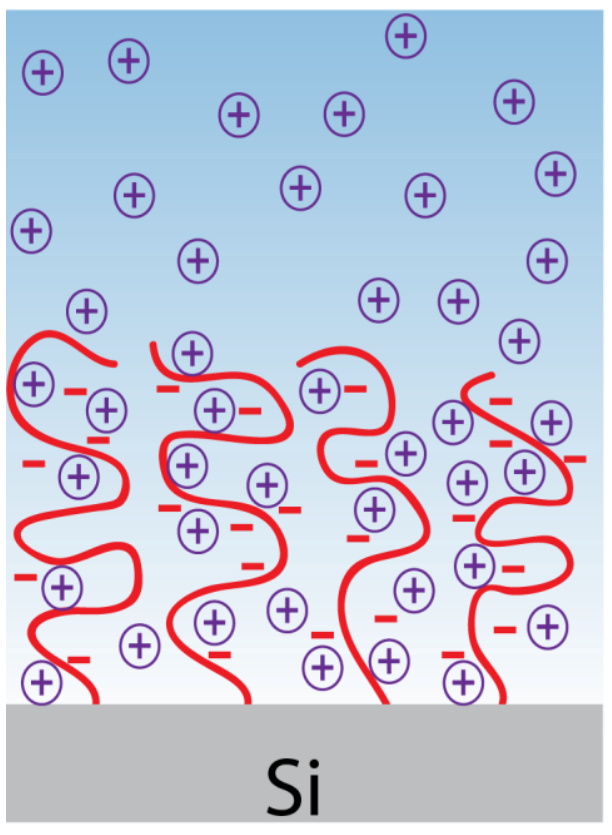

(b) Collapse

๑

$\oplus$

๑

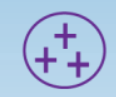

$\oplus$

๑

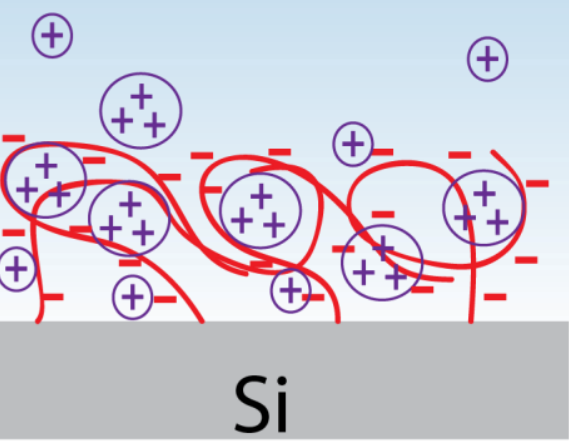

Figure 6 Through adjusting the external salt environment surrounding end-tethered polyelectrolyte chains, a polyelectrolyte brush can transition from an extended brush structure (a) to a collapsed structure (b).

\section{Conclusions}


The densely grafted PSS brush in this study retained its extended state in a media environment of $1 \mathrm{M}$ mono-valent $\mathrm{RbNO}_{3}$ salt. This stable brush structure is of great importance to a variety of applications, such as stabilizing colloidal particles and providing good boundary lubrication. The abrupt shrinkage of the brush thickness in the presence of tri-valent $\mathrm{Y}^{3+}$ cations highlights the effect of multi-valent counterions to the structure of polyelectrolyte brushes. We show here that these effects are highly relevant in dense brushes as they have previously been shown to be in sparse brushes. The work presented can have a significant impact in understanding the structure of polyelectrolyte brushes and developing functional polyelectrolyte brush systems using SI-ATRP.

\section{Acknowledgement}

This work was supported by the U.S. Department of Energy, Office of Science, Program in Basic Energy Sciences, Division of Materials Science and Engineering. We acknowledge the MRSEC Shared User Facilities at the University of Chicago supported by NSF Award NSF-DMR-1420709.

\section{References}

[1] de Gennes, P.G., Conformations of Polymers Attached to an Interface. Macromolecules, 1980. 13(5): p. 1069-1075.

[2] Ito Y, Nishi S, Park YS, Imanishi Y. Oxidoreduction-Sensitive Control of Water Permeation through a Polymer Brushes-Grafted Porous Membrane. Macromolecules, 1997. 30(19): p. 5856-5859.

[3] Toomey R, Tirrell M. Functional Polymer Brushes in Aqueous Media from Self-Assembled and Surface-Initiated Polymers. Annual Review of Physical Chemistry, 2008. 59(1): p. 493-517.

[4] Tugulu S, Arnold A, Sielaff I, Johnsson K, Klok H-A. Protein-Functionalized Polymer Brushes. Biomacromolecules, 2005. 6(3): p. 1602-1607. 
[5] Klein J, Kamiyama Y, Yoshizawa H, Israelachvili JN, Fredrickson GH, Pincus P, et al. Lubrication forces between surfaces bearing polymer brushes. Macromolecules, 1993. 26(21): p. 5552-5560.

[6] Raviv U, Giasson S, Kampf N, Gohy JF, Jerome R, Klein J. Lubrication by charged polymers. Nature, 2003. 425(6954): p. 163-165.

[7] Stuart MAC, Huck WTS, Genzer J, Muller M, Ober C, Stamm M, et al. Emerging applications of stimuli-responsive polymer materials. Nat Mater, 2010. 9(2): p. 101-113.

[8] Wang T, Long YC, Liu LD, Wang XW, Craig VSJ, Zhang GZ, et al. Cation-Specific Conformational Behavior of Polyelectrolyte Brushes: From Aqueous to Nonaqueous Solvent. Langmuir, 2014. 30(43): p. 12850-12859.

[9] Gong P, Wu T, Genzer J, Szleifer I. Poisson-Boltzmann Theory of pH-Sensitive (Annealing) Polyelectrolyte Brush. Langmuir, 2011. 27(17): p. 10615-10633.

[10] Gong P, Wu T, Genzer J, Szleifer I. Behavior of surface-anchored poly(acrylic acid) brushes with grafting density gradients on solid substrates: 2 . Theory. Macromolecules, 2007. 40(24): p. 8765-8773.

[11] Wu T, Gong P, Szleifer I, Vlcek P, Subr V, Genzer J. Behavior of surfaceanchored poly(acrylic acid) brushes with grafting density gradients on solid substrates: 1. Experiment. Macromolecules, 2007. 40(24): p. 8756-8764.

[12] Pincus, P., Colloid stabilization with grafted polyelectrolytes. Macromolecules, 1991. 24(10): p. 2912-2919.

[13] Balastre M, Li F, Schorr P, Yang J, Mays JW, Tirrell MV. A Study of Polyelectrolyte Brushes Formed from Adsorption of Amphiphilic Diblock Copolymers Using the Surface Forces Apparatus. Macromolecules, 2002. 35(25): p. 9480-9486.

[14] Li F, Balastre M, Schorr P, Argillier JF, Yang J, Mays JW, et al. Differences between Tethered Polyelectrolyte Chains on Bare Mica and Hydrophobically Modified Mica. Langmuir, 2006. 22(9): p. 4084-4091.

[15] Ahrens, H., S. Forster, and C.A. Helm, Charged polymer brushes: Counterion incorporation and scaling relations. Physical Review Letters, 1998. 81(19): p. $4172-4175$.

[16] Tran, Y., P. Auroy, and L.T. Lee, Determination of the structure of polyelectrolyte brushes. Macromolecules, 1999. 32(26): p. 8952-8964.

[17] Brettmann BK, Laugel N, Hoffmann N, Pincus P, Tirrell M. Bridging contributions to polyelectrolyte brush collapse in multivalent salt solutions. Journal of Polymer Science Part A: Polymer Chemistry, 2016. 54, 284-291.

[18] Israels R, Leermakers FAM, Fleer GJ, Zhulina EB. Charged Polymeric Brushes: Structure and Scaling Relations. Macromolecules, 1994. 27(12): p. 32493261.

[19] Zhulina EB, Borisov OV, Pryamitsyn VA, Birshtein TM. Coil Globule Type Transitions in Polymers .1. Collapse of Layers of Grafted Polymer-Chains. Macromolecules, 1991. 24(1): p. 140-149.

[20] Jiang, T. and J.Z. Wu, Ionic effects in collapse of polyelectrolyte brushes. Journal of Physical Chemistry B, 2008. 112(26): p. 7713-7720.

[21] Guptha, V.S. and P.Y. Hsiao, Polyelectrolyte brushes in monovalent and multivalent salt solutions. Polymer, 2014. 55(12): p. 2900-2912. 
[22] Hehmeyer OJ, Arya G, Panagiotopoulos AZ, Szleifer I. Monte Carlo simulation and molecular theory of tethered polyelectrolytes. Journal of Chemical Physics, 2007. 126(24).

[23] Mei Y, Hoffmann M, Ballauff M, Jusufi A. Spherical polyelectrolyte brushes in the presence of multivalent counterions: The effect of fluctuations and correlations as determined by molecular dynamics simulations. Physical Review E, 2008. 77(3).

[24] Schneider C, Jusufi A, Farina R, Li F, Pincus P, Tirrell M, et al. Microsurface Potential Measurements: Repulsive Forces between Polyelectrolyte Brushes in the Presence of Multivalent Counterions. Langmuir, 2008. 24(19): p. 10612-10615.

[25] Mei Y, Lauterbach K, Hoffmann M, Borisov OV, Ballauff M, Jusufi A. Collapse of Spherical Polyelectrolyte Brushes in the Presence of Multivalent Counterions. Physical Review Letters, 2006. 97(15): p. 158301.

[26] Farina R, Laugel N, Pincus P, Tirrell M. Brushes of strong polyelectrolytes in mixed mono- and tri-valent ionic media at fixed total ionic strengths. Soft Matter, 2013. 9(44): p. 10458-10472.

[27] Farina R, Laugel N, Yu J, Tirrell M. Reversible Adhesion with Polyelectrolyte Brushes Tailored via the Uptake and Release of Trivalent Lanthanum Ions. Journal of Physical Chemistry C, 2015. 119(26): p. 14805-14814.

[28] Hui CM, Pietrasik J, Schmitt M, Mahoney C, Choi J, Bockstaller MR, et al.Surface-Initiated Polymerization as an Enabling Tool for Multifunctional (Nano-)Engineered Hybrid Materials. Chemistry of Materials, 2014. 26(1): p. 745-762.

[29] Iddon PD, Robinson KL, and Armes SP, Polymerization of sodium 4styrenesulfonate via atom transfer radical polymerization in protic media. Polymer, 2004. 45(3): p. 759-768.

[30] Kienzle PA, O'Donovan KV, Ankner JF, Berk NF, Majkrzak CF; http://www.ncnr.nist.gov/reflpak. 2000-2006.

[31] Kienzle PA, Krycka J, Patel N, Sahin I. (2011). Refl1D (Version 0.7.7) [Computer Software]. College Park, MD: University of Maryland. Retrieved November 06, 2014. Available from http://reflectometry.org/danse

[32] Karim A, Douglas JF, Horkay F, Fetters LJ, Satija SK. Comparative swelling of gels and polymer brush layers. Physica B, 1996. 221(1-4): p. 331-336. 
Graphical Abstract

\section{Brush}

$\oplus \oplus$
$\oplus$
๑
$\oplus \oplus$
$\oplus$
$\oplus$
$\oplus$

†

$\overbrace{-\oplus}^{\oplus}$

(†)

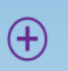

\section{Collapse}

†

†

†

$+$

Add $\mathrm{Y}^{3+}$

$\oplus$

$\oplus$

$\oplus$

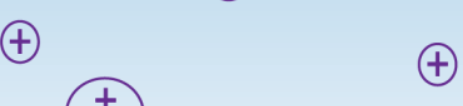

$\oplus$

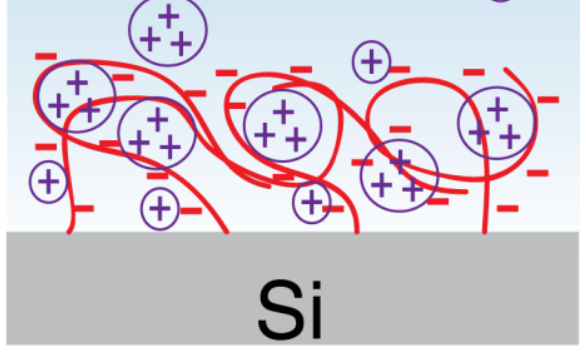

